Mon. Not. R. Astron. Soc. 000, 1-10 (2019) Printed 27 April $2020 \quad$ (MN LATEX style file v2.2)

\title{
Multi-wavelength observations of PSR J2032+4127 during the 2017 periastron passage
}

\author{
M. Chernyakova, ${ }^{1,2 \star}$ D. Malyshev ${ }^{3}$ P. Blay, ${ }^{4}$ B. van Soelen, ${ }^{5}$ S. Tsygankov ${ }^{6,7}$ \\ 1 School of Physical Sciences and CfAR, Dublin City University, Dublin 9, Ireland \\ 2 Dublin Institute for Advanced Studies, 31 Fitzwilliam Place, Dublin 2, Ireland \\ 3 Institut für Astronomie und Astrophysik Tübingen, Universität Tübingen, Sand 1, D-72076 Tübingen, Germany \\ 4 Valencian International University, Carrer del Pintor Sorolla, 21, 46002 Valencia, Valencia, Spain \\ 5 University of the Free State, Department of Physics, PO Box 339, 9300 Bloemfontein, South Africa \\ 6 Department of Physics and Astronomy, FI-20014 University of Turku, Finland \\ 7 Space Research Institute of the Russian Academy of Sciences, Profsoyuznaya Str. 84/32, Moscow 117997, Russia
}

Received $<$ date $>$; in original form $<$ date $>$

\begin{abstract}
PSR J2032+4127 is only the second known gamma-ray binary where it is confirmed that a young radio pulsar is in orbit around a Be-star. The interaction of the pulsar wind with the mass outflow from the companion leads to broad band emission from radio up to $\mathrm{TeV}$ energies. In the current paper we present results of optical monitoring of the 2017 periastron passage with the Nordic Optical Telescope. These observations are complemented by X-ray (Swift/XRT, NuSTAR) and GeV (Fermi/LAT) monitoring. Joint analysis of the evolution of the parameters of the $\mathrm{H} \alpha$ line and the broadband (X-ray to $\mathrm{TeV}$ ) spectral shape allows us to propose a model linking the observed emission to the interaction of the pulsar and Be-star winds under the assumption of the inclined disc geometry. Our model allows the observed flux and spectral evolution of the system to be explained in a self-consistent way.
\end{abstract}

Key words: pulsars: individual: PSR J2032+4127 - stars: emission-line, Be - X-rays: binaries - radiation mechanisms: non-thermal - methods: data analysis - methods: observational

\section{INTRODUCTION}

Gamma-ray binaries are a small, but growing class of high mass binary systems, which are characterized by nonthermal emission which peaks in the gamma-ray regime ( $>1 \mathrm{MeV}$, see e.g. Dubus 2013). To date there are eight known systems, all of which consist of an $\mathrm{O}$ or B types star and a compact object in the mass range of a neutron star or black hole (e.g. Chernyakova et al. 2019; Corbet et al. 2019; Dubus 2013). While the compact object is, for most systems, not directly detected, it is most likely a young, non-accreting radio pulsar and the non-thermal emission is produced in the shock that forms between the pulsar and stellar winds. This is known to be the case for two systems, namely PSR B125963 and PSR J2032+4127, where pulsed emission has been detected (Johnston et al. 1992; Abdo et al. 2009).

Shortly after the Fermi discovery of PSR J2032+4127 as $\mathrm{a} \approx 143 \mathrm{~ms}$ gamma-ray pulsar, it was also detected to be a pulsar at radio frequencies (Camilo et al. 2009). The source lies close to the extended TeV HEGRA source, $\mathrm{TeV}$ J2032+4130 (Aharonian et al. 2005), and it was

\footnotetext{
^ E-mail: masha.chernyakova@dcu.ie
}

proposed that this was a pulsar wind nebula powered by PSR J2032+4127 (Aliu et al. 2014). However, while PSR J2032+4127 was first thought to be an isolated pulsar (Camilo et al. 2009), further radio observations demonstrated a rapid increase in its observed spin-down rate, which was interpreted as evidence that the pulsar is a member of a highly-eccentric binary system, where the optical companion is a $\sim 15 \mathrm{M}_{\odot}$ Be star, MT91 213 (Lyne et al. 2015). PSR J2032+4127 thus turned out to be similar to PSR B1259-63, where a $48 \mathrm{~ms}$ pulsar is orbiting around a Be star, LS 2883, in an eccentric, 3.4 years orbit (e.g. Johnston et al. 1992).

Further multi-wavelength monitoring of PSR J2032+4127 by Ho et al. (2017) refined the orbital period to be in the range of 16000 to 17670 days, with an eccentricity of $e=0.961$ (separation at periastron is $\alpha_{\text {per }} \sim 1 \mathrm{au}$ ) and found the periastron passage would occur in November 2017. Subsequent observations around this period were in good agreement with this prediction, and in this paper we adopt $t_{\text {per }}=$ MJD 58069 as the date of the 2017 periastron.

Chandra X-ray observations reported in $\mathrm{Ho}$ et al. (2017) demonstrated a strong rise in the source brightness; 
PSR J2032+4127 was about 20 times brighter in 2016 than it was in 2010, and about 70 times brighter than it was in 2004. This increase was interpreted as a result of the collision between pulsar and Be star winds. Smoothed-particle hydrodynamics (SPH) modelling of the winds interaction presented in Coe et al. (2019) demonstrates the deformation and even partial destruction of the Be star disc at some orbital phases.

Modelling of the source suggested that around periastron the binary should be detectable at $\mathrm{TeV}$ energies (Bednarek, Banasiński \& Sitarek 2018; Takata et al. 2017) and PSR J2032+4127 was subsequently detected at TeV gamma-ray energies by the VERITAS and MAGIC telescopes as a point-like source (Abeysekara et al. 2018). The TeV light curve peaked at periastron (followed by a short dip a few days later). The observations also showed that during the brightest period (at periastron) the spectrum is best fit by a power-law but when the source was fainter during the 2017 observations (before periastron) a power-law with an exponential cut-off is favoured.

Long term observations of the Be optical companion, MT91 213, have shown the star has demonstrated strong variability showing periods of marked changes in the emission lines. Earlier observations (during 2012 and 2013) showed the stellar spectrum changed between a normal B star to a Be star (Salas et al. 2013) while Ho et al. (2017) showed that the $\mathrm{H} \alpha$ equivalent width varied from $W_{\lambda}=-13.2 \AA$ (in 2009) down to $W_{\lambda} \approx-4 \AA$ (in 2014-early 2016). It subsequently showed a rise to $W_{\lambda} \approx-10 \AA$ before decreasing again over $\mathrm{a} \sim 200$ day period in the second half of 2016 (Ho et al. 2017; Coe et al. 2019). Around $\sim 200 \mathrm{~d}$ before periastron, it is seen the equivalent width smoothly decreases towards periastron (Coe et al. 2019; Kolka et al. 2017; Rouco Escorial et al. 2019).

In this paper we present results of intensive optical observations of PSR J2032+4127 with the Nordic Optical Telescope (NOT) starting approximately 100 days before periastron and lasting up to 50 days after it. These observations were complemented by X-ray (Swift/XRT, NuSTAR) and $\mathrm{GeV}$ (Fermi/LAT) monitoring. Joint analysis of the evolution of the parameters of the $\mathrm{H} \alpha$ line and the broadband (X-ray to $\mathrm{TeV}$ ) spectral shape allow us to propose a model linking the observed emission to the interaction of the pulsar and Be-star winds.

\section{DATA ANALYSIS}

\subsection{Fermi/LAT data analysis}

Fermi/LAT data selected for the analysis presented in this paper cover more than 11 years (Aug. 2008 to Dec. 2019). We used the latest available fermitools with P8_R3 response functions (CLEAN photon class). ${ }^{1}$

To extract the spectra we performed the standard binned likelihood analysis of a region around PSR J2032+4127. The spectral analysis is based on the fitting of the spatial/spectral model of the sky region around the source of interest to the data. The region-of-interest considered in the analysis is a circle with a radius of 18 degrees around PSR J2032+4127. The model of the region included all sources from the 4FGL catalogue (The Fermi-LAT collaboration 2019) as well as components for isotropic and galactic diffuse emissions given by the standard spatial/spectral templates iso_P8R3_CLEAN_V2.txt and gll_iem_v07.fits.

The spectral template for each 4FGL source in the region was selected according to the catalogue model. The normalizations of the sources were considered to be free parameters during the fitting procedure. Following the recommendation of the Fermi/LAT collaboration, we performed our analysis with energy dispersion handling enabled.

\subsection{X-ray analysis}

\subsubsection{Swift/XRT}

We accompanied the analysis of the source behaviour with historic Swift/XRT observations of the region and the data from our recent Swift/XRT monitoring campaign of PSR J2032+4127. The data were reprocessed and analysed as suggested by the Swift/XRT team ${ }^{2}$ with the xrtpipeline v.0.13.4 and heasoft v.6.22 software package. The spectral analysis of Swift/XRT spectra was performed with XSPEC v.12.9.1m.

\subsubsection{NUSTAR}

Three NuSTAR observations of PSR J2032+4127 were performed on MJDs 57640, 58062 and 58078. The raw data were processed with the standard pipeline processing (HEASOFT v.6.22 with the NuSTAR subpackage v.1.8.0). We applied strict criteria for the exclusion of data taken in the South Atlantic Anomaly (SAA) and in the "tentacle"-like region of higher background activity near part of the SAA. Leveltwo data products were produced with the nupipeline tool with the flags SAAMODE=STRICT and TENTACLE=yes. Highlevel spectral products (spectra, response matrices, and auxiliary response files) were extracted for a point source with the nuproducts routine. The corresponding background flux was derived from a ring-like (inner/outer radii of 39" /86") region surrounding the source. The spectral analysis was performed in the energy range of $3-70 \mathrm{keV}$.

\subsection{Optical spectroscopy}

High resolution $(\mathrm{R}=25000)$ spectra were taken with the NOT from MJD 57950 to 58120, covering almost the whole periastron passage of the target. The instrument used was the FIber-fed Echelle Spectrograph (FIES, Telting et al. 2014 ) in its lower resolution mode. The spectra cover the range from 3800 up to $8300 \AA$. Exposure times were optimised for a good signal to noise ratio in the $\mathrm{H} \alpha$ line region, therefore the signal to noise in the blue bands is very low. This, together with the emission lines filling in all visible Balmer lines in that range, made the blue part of the spectra unusable for spectral characterisation of the target. A summary of the spectra and signal to noise ratio around the $\mathrm{H} \alpha$ line is shown in Table 1 . The history of $\mathrm{H} \alpha$ profiles is shown in Fig. 1. 

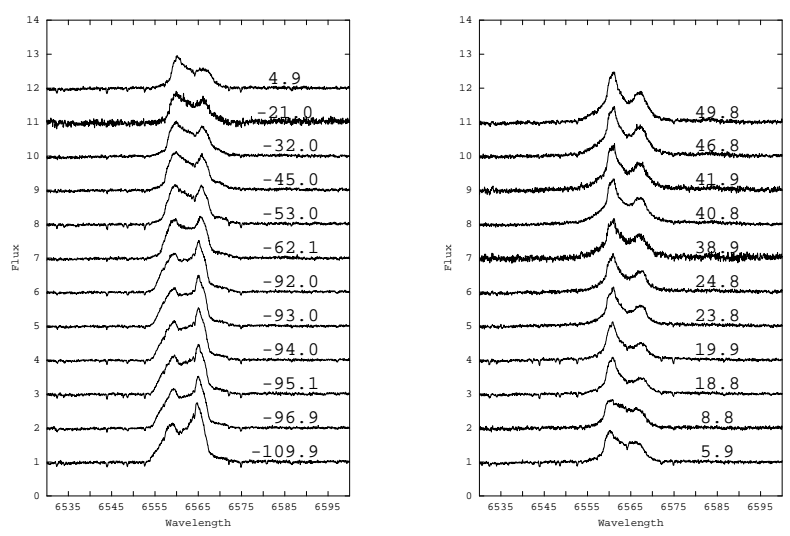

Figure 1. Evolution of the $\mathrm{H} \alpha$ line profile. Time runs upwards and from left to right. The difference (in days) between MJD and periastron passage (58069) is shown on the right side of each profile. Details for each spectrum are shown in Table 1.

Table 1. Summary of $\mathrm{H} \alpha$ parameters (namely, equivalent width in $\AA$, peak ratio and peak distance in $\mathrm{km} \mathrm{s}^{-1}$ ) are shown, together with the middle MJD time relative to periastron, signal to noise $(\mathrm{S} / \mathrm{N})$ ratio and exposure time (EXP.). The given $\mathrm{S} / \mathrm{N}$ refers to the continuum around the $\mathrm{H} \alpha$ line.

\begin{tabular}{ccccccc}
\hline MJD & MJD-T $T_{\text {per }}$ S/N & $\begin{array}{c}\text { EXP. } \\
(\mathrm{ks})\end{array}$ & $\begin{array}{c}\mathrm{EW} \\
(\AA)\end{array}$ & $\mathrm{V} / \mathrm{R}$ & $\begin{array}{c}\mathrm{R}-\mathrm{V} \\
\left(\mathrm{km} \mathrm{s}^{-1}\right)\end{array}$ \\
\hline 57959.15 & -109.9 & 50 & 2.5 & $-12.91 \pm 0.12$ & $0.66 \pm 0.01$ & $277 \pm 3$ \\
57972.14 & -96.0 & 50 & 2.7 & $-12.20 \pm 0.05$ & $0.78 \pm 0.03$ & $262 \pm 2$ \\
57973.95 & -95.1 & 50 & 2.7 & $-11.51 \pm 0.10$ & $0.76 \pm 0.03$ & $259 \pm 3$ \\
57975.00 & -94.0 & 50 & 2.7 & $-12.10 \pm 0.10$ & $0.75 \pm 0.02$ & $264 \pm 2$ \\
57976.05 & -93.0 & 50 & 2.7 & $-11.85 \pm 0.11$ & $0.81 \pm 0.05$ & $268 \pm 4$ \\
57977.05 & -92.0 & 50 & 2.7 & $-12.16 \pm 0.11$ & $0.74 \pm 0.03$ & $256 \pm 3$ \\
58006.90 & -62.1 & 60 & 1.2 & $-11.19 \pm 0.06$ & $0.95 \pm 0.05$ & $287 \pm 3$ \\
58016.04 & -53.0 & 45 & 2.5 & $-10.68 \pm 0.08$ & $1.02 \pm 0.05$ & $292 \pm 3$ \\
58023.98 & -45.0 & 50 & 1.2 & $-10.08 \pm 0.06$ & $1.11 \pm 0.02$ & $297 \pm 1$ \\
58036.99 & -32.0 & 50 & 1.2 & $-8.47 \pm 0.06$ & $1.12 \pm 0.03$ & $298 \pm 4$ \\
58048.00 & -21.0 & 25 & 1.2 & $-6.80 \pm 0.11$ & $1.32 \pm 0.13$ & $297 \pm 3$ \\
58073.89 & 4.9 & 55 & 1.2 & $-6.46 \pm 0.03$ & $1.90 \pm 0.09$ & $343 \pm 2$ \\
58074.91 & 5.9 & 55 & 1.2 & $-6.44 \pm 0.06$ & $1.67 \pm 0.14$ & $319.8 \pm 0.4$ \\
58077.83 & 8.8 & 35 & 1.2 & $-6.22 \pm 0.04$ & $1.32 \pm 0.10$ & $300 \pm 1$ \\
58087.81 & 18.8 & 65 & 1.2 & $-6.21 \pm 0.07$ & $2.48 \pm 0.27$ & $327.5 \pm 0.5$ \\
58088.93 & 19.9 & 55 & 1.2 & $-6.54 \pm 0.06$ & $2.40 \pm 0.09$ & $338 \pm 1$ \\
58092.84 & 23.8 & 60 & 1.2 & $-7.02 \pm 0.05$ & $1.92 \pm 0.27$ & $330 \pm 1$ \\
58093.80 & 24.8 & 40 & 1.2 & $-7.04 \pm 0.07$ & $1.72 \pm 0.11$ & $324 \pm 1$ \\
58107.85 & 38.9 & 20 & 1.2 & $-8.26 \pm 0.05$ & $1.71 \pm 0.14$ & $290 \pm 4$ \\
58109.81 & 40.8 & 50 & 1.2 & $-9.52 \pm 0.06$ & $1.67 \pm 0.05$ & $305 \pm 1$ \\
58110.89 & 41.9 & 30 & 1.2 & $-9.13 \pm 0.11$ & $1.74 \pm 0.16$ & $312 \pm 3$ \\
58115.82 & 46.8 & 35 & 1.2 & $-10.14 \pm 0.05$ & $1.61 \pm 0.13$ & $297 \pm 1$ \\
58118.81 & 49.8 & 40 & 1.2 & $-10.45 \pm 0.08$ & $1.67 \pm 0.03$ & $292 \pm 1$ \\
\hline & & & & & &
\end{tabular}

In our observations the $\mathrm{H} \alpha$ line profile is always doublepeaked. The reddest peak is more prominent in our first spectrum, but that situation reverses in our last spectrum, where the bluest peak is the dominant one. This transition from one dominant peak to another is typical in Be star discs and is the observational footprint of a density wave within the disc (see e.g. Telting et al. 1994).

We have performed a set of measurements of the $\mathrm{H} \alpha$ line which include the equivalent width (EW), the peak separation $\mathrm{R}-\mathrm{V}$, where $\mathrm{R}$ represents the red-most peak position

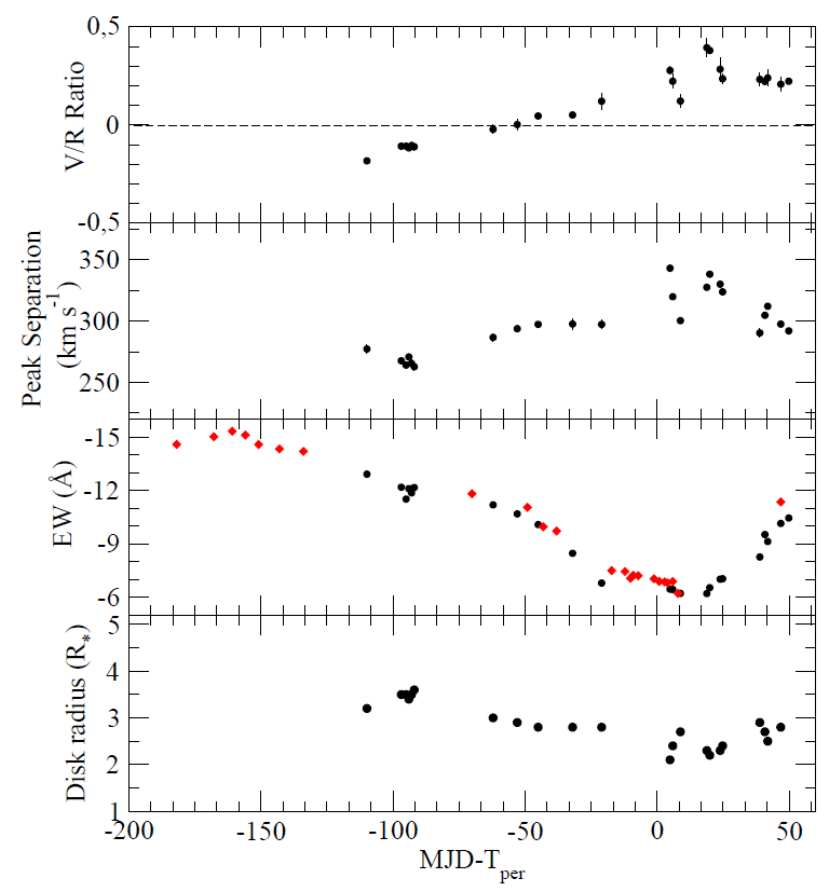

Figure 2. Evolution of the $\mathrm{H} \alpha$ parameters from the FIES spectra of MT91 213. Equivalent width measurements from Coe et al. (2019) are shown in red (without errors) for comparison; the $\mathrm{V} / \mathrm{R}$ ratio and peak separation are excluded because of the large errors. The lower panel shows the radius of the $\mathrm{H} \alpha$ emitting region of the disc.

and $\mathrm{V}$ the blue-most peak, and the ratio of peak fluxes $\mathrm{V} / \mathrm{R}$. In order to calculate the $\mathrm{R}-\mathrm{V}$ distance in $\mathrm{km} \mathrm{s}^{-1}$, we have used the relationship $(R-V)=c\left(\lambda_{R}-\lambda_{V}\right) / \lambda_{\alpha}$, where $c$ is the speed of light and $\lambda_{\alpha}$ is the rest wavelength of the $H_{\alpha}$ line. These parameters characterise the properties of the circumstellar discs around Be stars. The evolution in time of these three parameters can be seen in Fig. 2. Peak positions and fluxes have been obtained directly from the observed profile, by smoothing and finding each maximum when the slope of the line profile becomes null. All our measurements are in good agreement with those of Coe et al. (2019).

We see a decreasing EW as the neutron star approached its periastron. Right after the periastron passage, the disc becomes unstable and all three parameters show large variability. They recover a more steady trend afterwards, with the EW rising towards previous values and both the peak separation and ratio decreasing towards values similar to those before periastron passage. The high degree of variability after the periastron passage may be directly linked to the instabilities created by tidal interactions with the neutron star, as in previous epochs the reported variability is smoother and takes place over longer time scales (see Coe et al. 2019).

In order to look deeper into the detailed $\mathrm{H} \alpha$ line structure, we have selected the most symmetric profiles (those at MJD 58006 and MJD 58016, when the $\mathrm{V} / \mathrm{R}$ ratio is $\sim 1$ ), and after averaging them we have subtracted that average from the rest of spectra. The results are shown in Fig. 3. There we can see the excess in emission moving from the right (red side) towards the left (blue side). We also see some residuals at the line wings which evolve in the opposite direc- 
tion. These residuals at the wings are a consequence of the $\mathrm{H} \alpha$ line asymmetry. This asymmetry is expected for density waves perturbing the disc (Hummel \& Hanuschik 1997) and are dependent on the inclination of the disc with respect to the line of sight. The asymmetric profile in $\mathrm{H} \alpha$, according to Hummel \& Hanuschik (1997), would correspond to a disc inclination angle of between 25 and 50 degrees.

The peak of the excess in emission responsible of the $\mathrm{V} / \mathrm{R}$ variability (Fig. 3) moves from a redder $\left(t_{\text {per }} \sim\right.$ $-109.9 \mathrm{~d})$ to a bluer $\left(t_{\mathrm{per}} \sim 49.8 \mathrm{~d}\right)$ position by a distance on the order of $\sim 170 \mathrm{~km} \mathrm{~s}^{-1}$. The peak separation shown in Fig. 2, with an average of $\sim 290 \mathrm{~km} \mathrm{~s}^{-1}$, is also influenced by the emission excess in the line wings. The bluest peak of this wing excess at $t_{\text {per }} \sim-109.9 \mathrm{~d}$ differs in position with respect to the reddest one at $t_{\text {per }} \sim 49.8 \mathrm{~d}$ on the order of $\sim 560 \mathrm{~km} \mathrm{~s}^{-1}$. This distance is in agreement with what is expected from the model of Hummel \& Hanuschik (1997).

The lower panel on Fig. 2 shows the evolution of the circumstellar disc radius. In order to estimate the disc radius, first we have estimated the projected rotational velocity of MT91 213 from the peak separations (R-V) seen in Table 1 by using the relationship

$$
\Delta \text { peak } \sim(1.2 \pm 0.6) v_{\text {rot }} \sin i
$$

which is valid for $\mathrm{H} \alpha$ EWs lower than $15 \AA$ (Hanuschik 1989). We obtain a projected rotational velocity of $250 \pm$ $20 \mathrm{~km} \mathrm{~s}^{-1}$. With this projected rotational velocity, and using the relationship (see, e.g., Reig et al. 2016)

$$
\frac{R_{\mathrm{disc}}}{R_{*}}=\left(\frac{2 v_{\mathrm{rot}} \sin i}{\Delta \text { peak }}\right)^{1 / j}
$$

we have calculated $R_{\text {disc }} / R_{*}$. The $j$ in the exponent is expected to be $\sim 0.5$ for a Keplerian disc (Reig et al. 2016). By fitting

$$
\log \left(\frac{\Delta \text { peak }}{v_{\text {rot }} \sin i}\right)=-\left(\frac{j}{2}\right) \log \left(-\mathrm{EW}_{\mathrm{H} \alpha}\right)+b
$$

(see Reig et al. 2016) to the measurements from all our spectra we obtained $j \sim 0.53$.

\section{RESULTS AND DISCUSSION}

\subsection{Optical evidence of the Be-star disc evolution.}

The optical behaviour of PSR J2032+4127 was previously discussed by e.g. Coe et al. (2019); Rouco Escorial et al. (2019); Kolka et al. (2017). However the majority of these observations occurred before periastron and the cadence of observations after periastron was much less. The NOT observations presented in the paper, complemented by the previous observations, show the behaviour of the disc until $\sim 50 \mathrm{~d}$ after periastron.

The observations of the $\mathrm{H} \alpha$ line in PSR J2032+4127 show a clear variability of the Be star disc, both in the change in equivalent width, and the $\mathrm{V} / \mathrm{R}$ profile. The equivalent width well away from periastron is $\left|W_{\lambda}\right|<9 \AA$ (Coe et al. 2019) but becomes stronger $\sim 181 \mathrm{~d}$ before periastron $\left(\left|W_{\lambda}\right| \sim 15 \AA\right)$. The EW then decreases towards periastron reaching a minimum $\left(\left|W_{\lambda}\right| \approx 6.2 \AA\right)$ a few days after periastron, while the $\mathrm{V} / \mathrm{R}$ profile shows a corresponding shift to a stronger blue component and an increase in the peak separation (Fig.1).

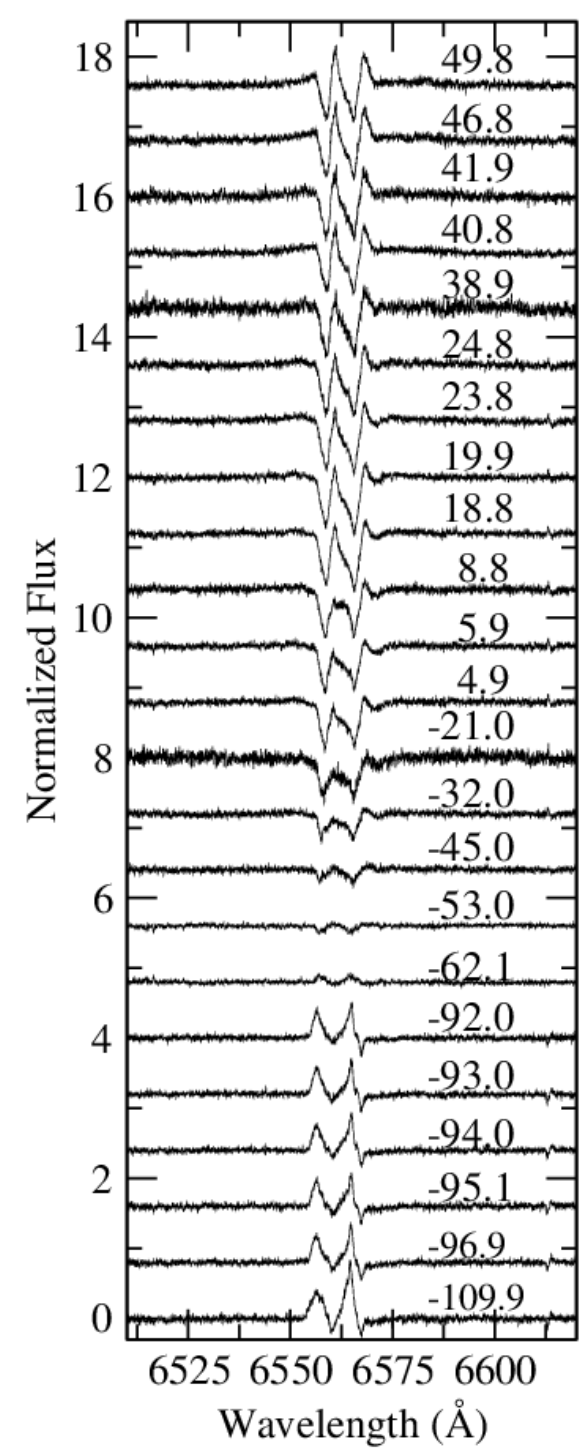

Figure 3. Difference between all spectra in Fig. 2 and the average of the two most symmetric ones, i.e., those for which $\mathrm{V} / \mathrm{R}$ ratio is $\sim 1$. We see the emission excess on the wings of the line due to line asymmetry, initially on the blue side but decreasing in intensity as the $\mathrm{V} / \mathrm{R}$ ration increases. This excess appears on the red side when $\mathrm{V} / \mathrm{R}>1$ and increases in intensity afterwards. The line wing at the side of the dominant peak falls more abruptly to the continuum than the wing at the opposite side. The excess due to zones of enhanced density propagating on the disc (the cause of the $V / R$ variability), seen in the central zone of these profiles, moves from red to blue.

The behaviour observed immediately around and after periastron is easily explained by the introduction of tidal interaction with the pulsar (see the SPH modelling in Coe et al. 2019). However, the behaviour of the disc before and leading up to periastron is more challenging.

Be stars are known to show intrinsic variability of the disc structure (see e.g. Rivinius, Carciofi \& Martayan 2013, and references therein), and a number of the observed changes in the disc, particularly seen far from periastron, are due to the intrinsic variability of the Be star and not the interaction with the pulsar. However, the clear variation of 
the EW towards periastron, with the line strength decreasing towards, and increasing after, periastron is very suggestive that this can be attributed to the interaction in the binary system. It should be noted that Kolka et al. (2017) suggested that the behaviour of the disc around periastron was due to intrinsic variation of the star and that size of the disc remained stable as they did not measure a shift in the position of the peaks of the $\mathrm{H}$ alpha line. However, the longer period and higher resolution observations presented here and in Coe et al. (2019) do find a shift.

While the optical emission occurs close to the Be star (see Fig. 2) the disc can be much larger. For example, Klement et al. (2017) suggested truncation radii of $\sim 100 R_{*}$, for some Be stars, which they attributed to undetected binary companions. In the viscous decretion disc model the density of Be disc decrease with radial distance $\rho \propto r^{-n}$, and this rate is lower of Be stars in binaries due to the accumulation of material within the truncation radius, resulting in denser discs (e.g. Zamanov et al. 2001; Okazaki et al. 2002; Panoglou et al. 2016). The highly eccentric nature of this binary may mean that as the pulsar approaches the star truncation of the disc slows the rate at which material can flow out of the decretion disc, causing an accumulation in material close to the star, increasing the EW. This may explain the rise in equivalent width from $\left|W_{\lambda}\right|<9 \AA$ to $\left|W_{\lambda}\right| \sim 15 \AA$ between the observations before $t_{\text {per }}-393 \mathrm{~d}$ and the next after $\sim t_{\text {per }}-181 \mathrm{~d}$ shown in Coe et al. (2019) (where $t_{\text {per }}$ is the time of periastron). There is also a clear change in the line profile with red peak significantly increasing (see fig. 5 in Coe et al. 2019). The gravity of the pulsar could further be causing a preferential direction for the disc outflow. However, it should be noted that at these times the binary separation is still large and underlying intrinsic variability of the Be star be driving this increase (Kolka et al. 2017).

The observations after $\sim t_{\text {per }}-181 \mathrm{~d}$ (in Coe et al. 2019, and this paper; see Fig. 2) show that the equivalent width peaks at $\sim t_{\text {per }}-161 \mathrm{~d}$ and then steadily decreases towards periastron. The NOT observations (starting from $\sim t_{\text {per }}-110 \mathrm{~d}$ ) also show a general increase in the peak separation. Since Be stars are believed to be Keplerian (e.g. Rivinius, Carciofi \& Martayan 2013) the peak separation traces the dominant or average $\mathrm{H} \alpha$ emission region, and the change suggests the emission region is moving closer to the Be star. This could be occurring because as the truncation of the disc increases, the emitting region becomes smaller, decreasing the EW and moving the average emission region slightly closer to star. The disc truncation could be induced by tidal effects, but additionally the pulsar wind may be blowing away regions of the disc. This behaviour is very different to the case of PSR B1259-63 where the equivalent width continues to grow past periastron. However, the typical equivalent width of the $\mathrm{H} \alpha$ line observed from PSR B1259-63 is much higher $\left(\left|W_{\lambda}\right| \sim 50 \AA\right)$ which suggests a much denser disc, than in the case of PSR J2032+4127.

The influence of the pulsar is also seen in the change in the $\mathrm{V} / \mathrm{R}$ profile. While the double peaked line was symmetric $(V / R \approx 1)$ far from periastron $\left(t_{\text {per }}-600\right.$ to $t_{\text {per }}-400 \mathrm{~d}$; Coe et al. 2019), after $\sim t_{\text {per }}-182 \mathrm{~d}$ the line is asymmetric and varying from redder to bluer. The variation in $V / R$ profile is normally attributed to density waves in the decretion disc, which in this case can be induced by the tidal interaction as is seen in SPH modelling of Be binary system (Okazaki et al. 2002; Panoglou et al. 2016; Coe et al. 2019). The variation in the $V / R$ ratio changes relatively smoothly as the pulsar approaches periastron. However, immediately after periastron (within a few ten of days) the variation in the $\mathrm{V} / \mathrm{R}$ profile and peak separation changes much more rapidly (Fig. 2). The peak separation reaches a maximum of $343 \mathrm{~km} \mathrm{~s}^{-1}$ before decreasing by $\sim 40 \mathrm{~km} \mathrm{~s}^{-1}$ in $\sim 3 \mathrm{~d}$, before increase by $\sim 40 \mathrm{~km} \mathrm{~s}^{-1}$ within $\sim 2 \mathrm{~d}$, before decreasing again. At the same time the $\mathrm{V} / \mathrm{R}$ profile first becomes slightly redder before becoming bluer again. Because the system is highly eccentric, the binary separation at periastron is $\sim 1$ au $\left(\sim 28 R_{\star}\right)$, and the pulsar will introduce a strong turbulence to the disc as well as an asymmetry to the disc structure. As the pulsar passes periastron more turbulent structures will be introduced into the disc and material can be pulled in the direction of the pulsar, then continuing to move towards us, resulting in a stronger blue component. The disc will become less symmetric and change more rapidly, causing the faster change in the $V / R$ ratio and peak separation. This basic structure is seen in the SPH models presented in Coe et al. (2019) where the disc becomes asymmetric and material is carried away from the disc.

During the post-periastron period the equivalent width starts to increase and we interpret this as the disc starting to increase in size as the binary separation increases.

\subsection{High Energy Emission}

While the optical emission detected from PSR J2032+4127 is mainly coming from the Be star and its disc, the observed $\mathrm{X}$-ray emission is usually attributed to the synchrotron emission of the relativistic electrons originating from the pulsar wind. The first X-ray detection of this system was in 2002 by Chandra (Camilo et al. 2009; Ho et al. 2017), which reported the detection of a new, very faint X-ray source at the position of PSR J2032+4127. Subsequent observations with Chandra and Swift/XRT demonstrated a steady flux rise as the pulsar approached periastron. The dependencies of the X-ray flux and slope on time are summarized in Fig. 4. The flux demonstrates a complicate behaviour with a sudden narrow dip close to periastron. The slope of the X-ray band spectrum hardens as the pulsar approaches the Be star, reaching a value of $\Gamma \sim 1.5$ and remains constant around the periastron passage, see also Pal et al. (2019) for the discussion of the spectral slope behaviour around the periastron. Note that no significant variation of the hydrogen column density was detected and for the presented analysis it was fixed to its mean value over the orbit, $N_{H}=0.884 \cdot 10^{22} \mathrm{~cm}^{-2}$. The correctness of the X-ray slopes measured by Swift/XRT (which can be biased due to a correlation with $N_{H}$ ) is supported by $N u S T A R$ measurements. These measurements performed at $>3 \mathrm{keV}$ energies are minimally affected by the absorption and are in a good agreement with the Swift/XRT results.

Contrary to the X-ray band, the flux and the spectral slope of PSR J2032+4127 remained stable in the GeV band for the whole observational period including the periastron passage, see Li et al. (2018) and Fig. 5.

Previously, the time evolution of PSR J2032+4127 was modelled by Li et al. (2018) (radio, X-ray and GeV bands) and Coe et al. (2019)(optics and X-ray). Li et al. (2018) sug- 

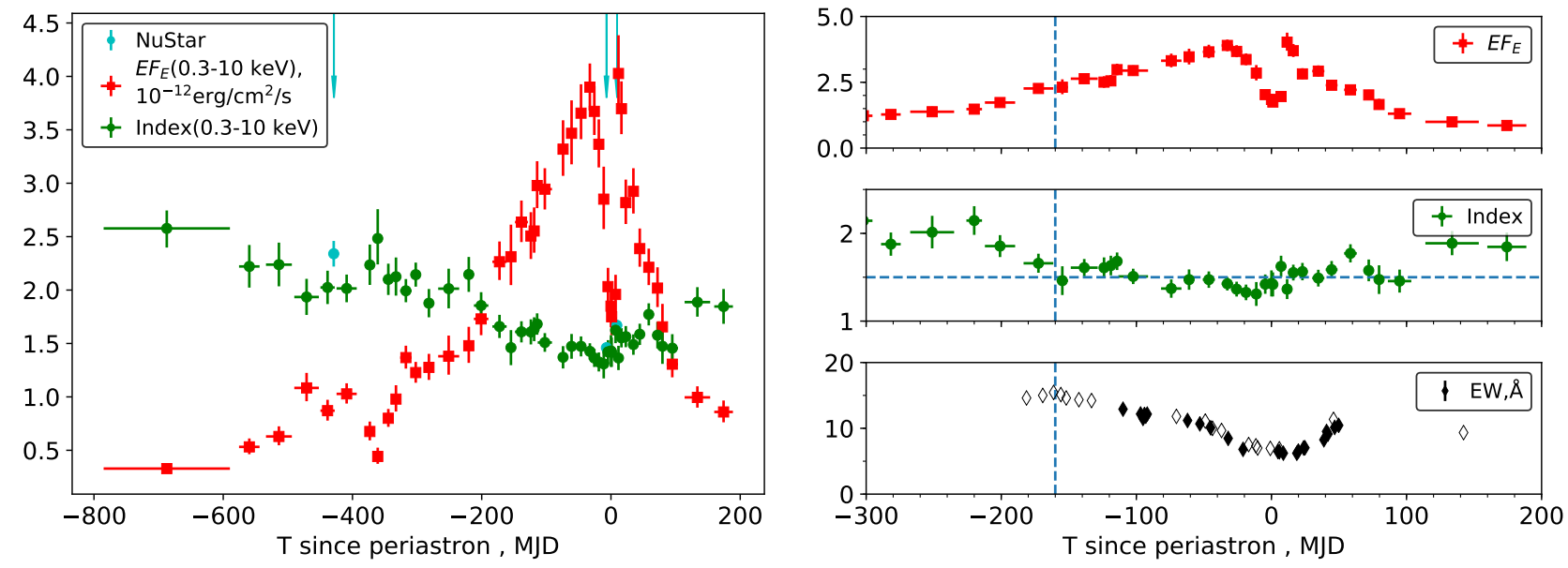

Figure 4. Swift/XRT 0.3-10 keV lightcurve of PSR J2032+4127 (red squares) and the corresponding slope (green circles). Cyan points and arrows demonstrate index $(3-70 \mathrm{keV})$ and periods of the $N u S T A R$ observations. Black symbols in the right panel illustrate the equivalent width of the $\mathrm{H} \alpha$ line reported in this paper (filled symbols) and Coe et al. (2019) (empty symbols). Vertical dashed line denotes the period of the peak of $\mathrm{H} \alpha$ equivalent width. Horizontal dashed line corresponds to the value of the X-ray slope equal to 1.5 .

gested that the strong X-ray dip close to periastron is explained by an increase of the magnetization parameter of the star-pulsar colliding winds shock accompanied by flux suppression due to Doppler boosting effect. The post-periastron rise could be a consequence of the Be stellar disc passage by the pulsar. The absence of variability in the $\mathrm{GeV}$ emission was explained by the strong dominance of the pulsar magnetospheric emission over the expected orbital-modulated Inverse Compton (IC) emission. The model was able to predict the overall shape of the orbital X-ray lightcurve, but was not able to reproduce the details of the double-peak flux structure around periastron. The origin of the hardening of the X-ray spectrum, as the pulsar approaches periastron, also does not appear in the model in a natural way and was attributed by the authors to a possible increase of the hydrogen column density. ${ }^{3}$

Coe et al. (2019) reported on optical and X-ray flux measurements of PSR J2032+4127, accompanied by SPH modelling of the Be star/pulsar interaction. In their model the authors explicitly assumed that the disc of the Be star is inclined to the orbital plane. The modelling, however, failed to describe the details of the observed X-ray lightcurve of the system, generally predicting a maximum of the flux at periastron and does not consider the variability of the X-ray spectrum.

Additionally, $\mathrm{Ng}$ et al. (2019) recently presented radio to X-ray observations of PSR J2032+4127. The authors suggested a potential spectral break at $\sim 5 \mathrm{keV}$ energies which was attributed to the modification of the spectrum of accelerated electrons by synchrotron losses without detailed modelling.

Below we propose a simple model for the observed emission from PSR J2032+4127 which allows the observed multiwavelength evolution of this system to be qualitatively explain. Within this model, similar to Coe et al. (2019), we

\footnotetext{
3 Please note, that only the hardness ratio was evaluated by Li
} et al. (2018) . suggest that the disc of the Be star is inclined to the orbital plane.

We would like to note that the inclination of the disc to the orbital plane was also proposed for other gammaray binary systems, e.g. PSR B1259-63 (Melatos, Johnston \& Melrose 1995; Chernyakova et al. 2006) and HESS J0632+057 (Malyshev et al. 2017). These systems demonstrate similar lightcurve with two peaks in X-ray band separated by a dip at periastron and are characterised by a long (longer than a year) orbital period. Such similarities allow us to speculate, that the "inclined disc" geometry can be typical for long-period Be-star hosting gamma-ray binaries. Inclined disc models have also been proposed for Be/X-ray binary systems (see e.g. Brown et al. 2019, and references therein). The reason for this could be that the systems are either too young or that the compact object spends too little time near the optical star to allow tidal forces to align the orbit of a compact source.

\subsubsection{Spectrum formation}

Similar to previous works (see e.g. Takata et al. (2017)) we propose that the $\mathrm{X}$-ray and $\mathrm{TeV}$ emission observed from PSR J2032+4127 originates from synchrotron and inverse Compton (IC) processes, respectively. The X-ray photon slope of $\Gamma \sim 1.5$ allows the slope of corresponding electron spectrum to be estimated as $\Gamma_{e} \sim 2$. At the same time the level of unpulsed radio emission reported by $\mathrm{Ng}$ et al. (2019) does not allow a simple continuation of $\Gamma \sim 1.5$ from radio up to X-ray energies and thus suggests the presence of a lowenergy feature (break or a cut-off) in the electron spectrum. In what follows below we discuss two possible mechanisms for the formation of such a spectrum, namely the cooling and acceleration scenarios.

Cooling scenario: close to mono-energetic electrons $\left(E_{e} \sim\right.$ $20 \mathrm{TeV}$ ) are continuously injecting by the pulsar wind at the pulsar-stellar wind interaction region. Continuous synchrotron and IC losses lead to the formation of the modified 


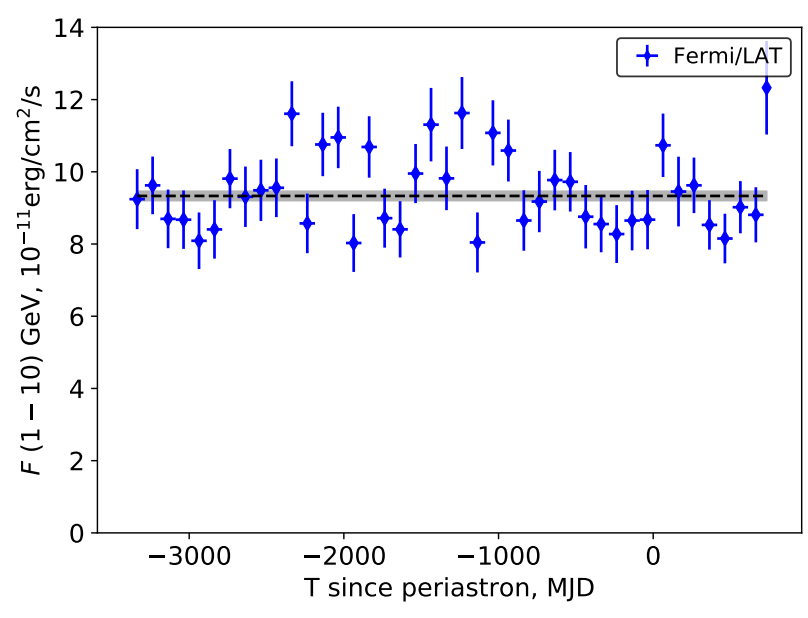

Figure 5. Fermi/LAT lightcurve of PSR J2032+4127 (1-10 $\mathrm{GeV}$ ) with a 100 days bins. The black dashed line and shaded region illustrate the best-fit of the data with a constant and the corresponding $1 \sigma$ uncertainty area.

electrons spectrum and subsequently the observed X-ray and $\mathrm{TeV}$ emission.

Acceleration scenario: similarly to the cooling scenario mono-energetic electrons are injected, but the energy is much lower $\left(E_{e} \sim 0.1 \mathrm{TeV}\right)$. The injected electrons accelerate at the pulsar-stellar winds interaction shock on a characteristic timescale

$$
t_{\mathrm{acc}} \approx 0.1\left(E_{e} / 1 \mathrm{TeV}\right) \eta\left(B_{0} / 1 \mathrm{G}\right)^{-1} \mathrm{~s}
$$

where $B_{0}$ is a magnetic field at the shock and $\eta \geqslant 1$ is the acceleration efficiency (see e.g. Khangulyan, Aharonian \& Bosch-Ramon 2008, for the details).

For typical magnetic field values considered for gammaray binaries, this timescale is much shorter than the synchrotron and IC loss timescales for the electrons with energies $\lesssim 100 \mathrm{TeV}$. The acceleration of the electrons can lead to the fast formation of a power-law (with a cut-off) electron spectrum with a slope of $\Gamma_{e} \sim-2$ (typical for Fermi acceleration) and a cut-off at $\sim 30 \mathrm{TeV}$ energies. The accelerated particles escape from the system on a timescale of $t_{e s c} \gtrsim d_{*} / c \sim 500 \mathrm{~s}$. This approach is similar to the one used by Takata et al. (2017).

The left panel of Fig. 6 illustrates the cooling times for electrons due to synchrotron and IC (using the approximation of Khangulyan, Aharonian \& Kelner 2014) emission for the parameters typical for Be-star gamma-ray binary systems (magnetic field $B_{0}=0.2 \mathrm{G}$; distance from the star to the emission region $d_{*}=0.7 \mathrm{au}$ ). The red dot-dashed line shows the acceleration time (Eq. 4) and horizontal black dotdashed line shows the escape time from the system (selected to be of the order of $10^{3} \mathrm{~s}$ ), while the dash blue and solid green lines show the synchrotron and IC cooling times, respectively. For these representative parameters it can be seen that the synchrotron and IC cooling timescales are longer than the escaping time at low energy $\left(E_{e}<5 \times 10^{12} \mathrm{eV}\right)$ and do not modify the electron's spectrum. However, at higher energies the synchrotron cooling time is short enough to substantially modify the injected spectrum (see the right panel of Fig. 6).

The electron spectrum for both the cooling and acceler- ation scenarios is determined by numerically calculating the synchrotron and IC losses of a continuously injected spectrum of electrons, until a steady solution is obtained. The electrons are assumed to be injected with a mono-energetic (cooling scenario) or a power-law with an exponential cutoff (acceleration scenario) spectra. The resulting electron spectra are shown in the right panel of Fig. 6.

\subsubsection{Spectral modelling}

As the pulsar start to approach to periastron $\left(t_{\text {per }}-600 \mathrm{~d}\right)$ the interaction of the pulsar wind with the wind of the Be star shifts the emission region towards the pulsar. The subsequent increase of the magnetic field in the emission region naturally explains the observed rise of the X-ray flux.

The optical observations clearly demonstrates the influence of the pulsar on the state of the Be-star disc. The interaction of the pulsar wind with the disc of the Be star leads to the rise of the X-ray emission. At $t_{\mathrm{per}}-160 \mathrm{~d}$ the equivalent width of the $\mathrm{H} \alpha$ line reaches its maximum and at around the same time the X-ray photon index becomes equal to $\Gamma=1.5$. Such a value can be expected in either the case of acceleration in a strong shock (acceleration scenario) (e.g. Jones 1994; Bell 2013) or effective cooling (cooling scenario) (e.g. Blumenthal \& Gould 1970). This slope value remains almost constant up to hundred days after periastron.

The rapid strong decrease of the flux close to periastron $\left(t_{\text {per }}-20\right.$ to $\left.t_{\text {per }}+10 \mathrm{~d}\right)$ corresponds to the pulsar entering a sparser regions due to the inclined Be-star disc. This consequently shifts the wind-wind interaction region further away from the pulsar and decreases the magnetic field in the emission region. The shift of the emission region closer to the star can also explain the evolution of the observed $\mathrm{TeV}$ emission. This emission is produced by the IC mechanism from the same population of electrons which form the $\mathrm{X}$-ray component of the observed spectrum. Contrary to Xray light curve there is no dip at periastron, but it rather demonstrates an increase in flux. This is in line with the proposed model, as the shift of the emission region towards the Be star implies an increase in the density of the soft photons and consequently an increased level of $\mathrm{TeV}$ emission reported by Abeysekara et al. (2018). The drop of the $\mathrm{TeV}$ flux immediately after the periastron is roughly coincident with second X-ray maximum and can be attributed to the attenuation of Be star's photons by dense regions of the disk.

Takata et al. (2017) studied details of the X-ray and $\mathrm{TeV}$ light curves in the scenario similar to the acceleration one. This work was done before the actual periastron passage took place and lacked knowledge of system parameters. This model didn't consider the possibility of the disc inclination and attributed two peaks in the X-ray light curve to the interplay between the orbital dependence of magnetisation parameter and Doppler boosting. Subsequent observations demonstrated that the model should be adjusted to explain the observed shape and positions of X-ray peaks.

The GeV emission observed by Fermi/LAT is stable along the whole orbit (see Fig. 5) and in our model this is produced by the pulsed magnetospheric emission from the pulsar. The quality of the data doesn't allow to observe the variability of the $\mathrm{GeV}$ emission predicted by Takata et al. (2017). 

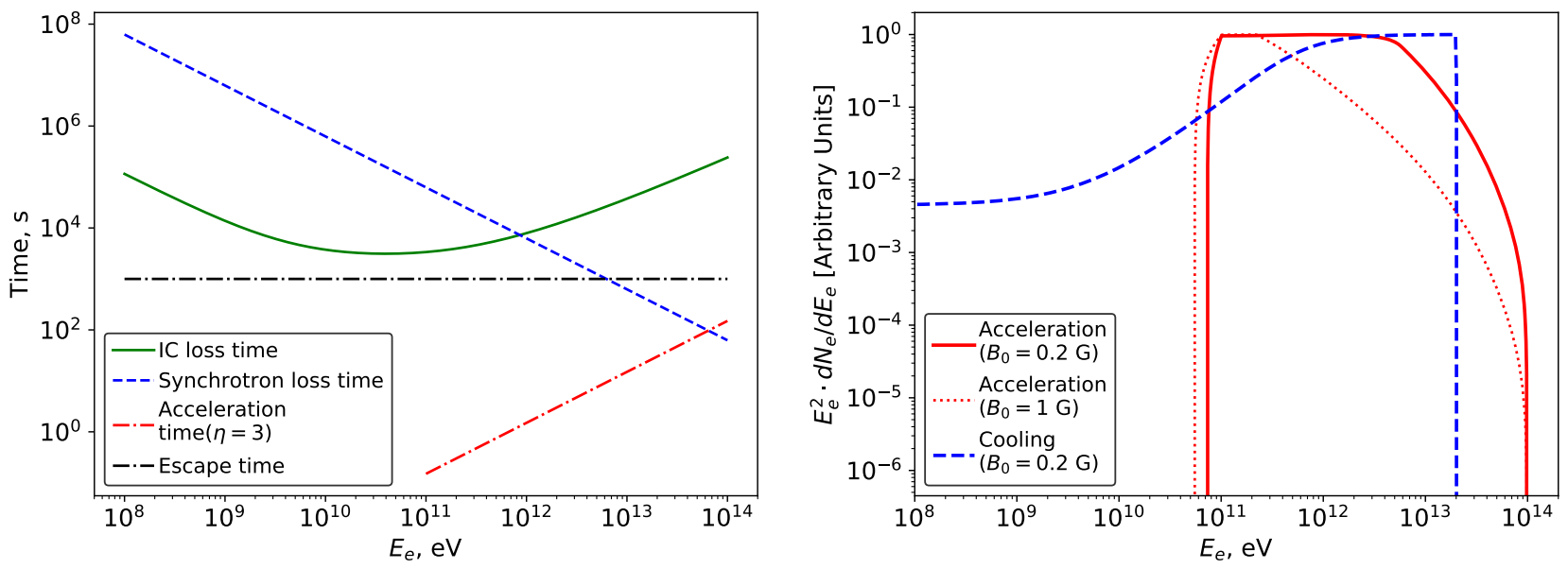

Figure 6. Left: Synchrotron and IC cooling times along with characteristic acceleration timescale for electrons. Right: Spectra of electrons produced by the "cooling" and "acceleration" scenarios.

The X-ray to TeV SED of the system is shown in Fig. 7 along with the results of spectral modelling of the system. Green symbols illustrate the available data close to periastron: VLA (triangles; $\mathrm{Ng}$ et al. (2019)), NuSTAR (squares), Fermi/LAT (circles), VERITAS and MAGIC(semi-transparent areas; Abeysekara et al. (2018)). The green semi-transparent diamond points show the NuSTAR spectrum taken at $\sim-400$ days before the periastron passage. The solid and dashed curves present the results of broad-band spectral modelling of the system for the "cooling" and "acceleration" scenarios.

The synchrotron and IC emission was calculated with the naima v.0.8.3 package (Zabalza 2015), which uses the analytic approximations for the IC and synchrotron emission developed by Aharonian \& Atoyan (1981); Aharonian, Kelner \& Prosekin (2010); Khangulyan, Aharonian \& Kelner (2014). The solid red line in Fig. 7 shows the result for the "acceleration" scenario and corresponds to an emitting region located at a mean distance of $d=0.7$ au from the star, a magnetic field strength of $B_{0}=0.2 \mathrm{G}$ and an escape time from the system of $t_{e s c}=10^{3} \mathrm{~s}$. The spectral modelling of the "cooling" scenario (blue dashed curves) for the same location of the emitting region requires a slightly higher magnetic field $\left(B_{0}=1 \mathrm{G}\right)$, and does not suggest the escape of electrons from the system. For both scenarios we assume the star to have a luminosity of $L_{*}=6 \cdot 10^{37} \mathrm{erg} / \mathrm{s}$ and a temperature $T_{*}=3 \cdot 10^{4} \mathrm{~K}$. The presented spectra directly correspond to the electron spectra shown in right panel of Fig. 6.

\subsection{Comparison to other "inclined disc" systems}

While the "inclined disc" geometry proposed for PSR J2032+4127 would suggest that it should be very similar to other gamma-ray binaries (e.g. PSR B1259-63 or HESS J0632+057), the system's behaviour during the periastron passage does not exactly resembles the behaviour of these other systems. While, similar to PSR B1259-63 and HESS J0632+057, PSR J2032+4127 demonstrates a double-peaked X-ray lightcurve with a deep minimum at periastron, contrary to these systems there is no hints of

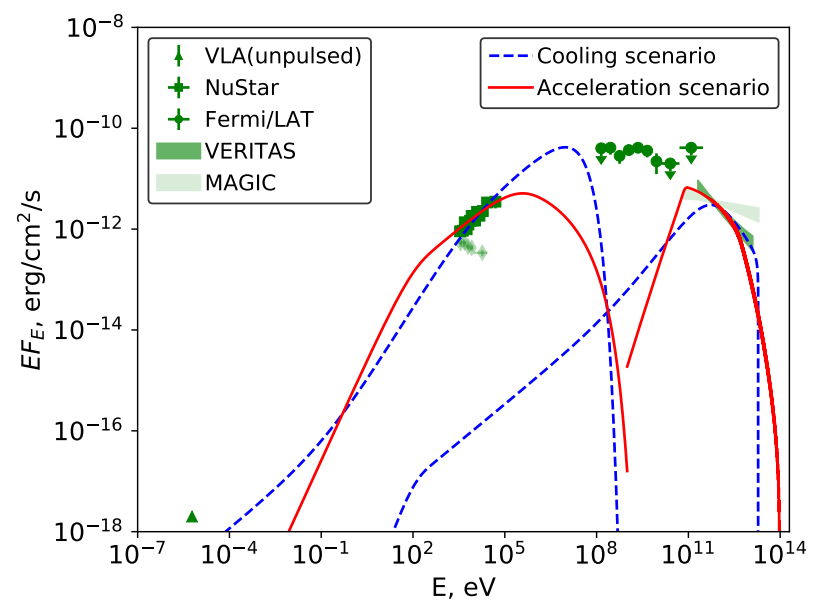

Figure 7. The radio to TeV spectrum of PSR J2032+4127. The green symbols illustrate the spectrum of the source close to periastron: VLA(triangles) Fermi/LAT (circles), NuSTAR (squares), VERITAS and MAGIC(semi-transparent regions). The green diamonds show the NUSTAR spectrum of PSR J2032+4127 measured at $\sim-400$ days before to periastron. The curves show the results of the broad-band spectral modelling of the system for the "cooling" (blue dashed) and acceleration (red solid) scenarios; see text for details. The radio and $\mathrm{TeV}$ data are from $\mathrm{Ng}$ et al. (2019); Abeysekara et al. (2018).

orbital variations of the hydrogen column density. The orbital X-ray spectral variability (which shows significant spectral hardening close to periastron) is also different from PSR B1259-63 (softening of the spectrum close to periastron, Chernyakova et al. 2015) and HESS J0632+057 (constant slope, Malyshev et al. 2017).

In the optical band, the differences in the behaviour of the $\mathrm{H} \alpha$ emission line, between PSR J2032+4127 and PSR B1259-63 systems are clearly seen. In contrast to what is observed in PSR B1259-63 (see Chernyakova et al. 2015) where the EW (which is linked to the disc size) increases as the neutron star approaches periastron, our data set, and that of Coe et al. (2019), shows the EW reaches a maximum 
about $180 \mathrm{~d}$ before periastron. But afterwards, in response to the proximity of the compact companion the disc size decreases again to levels similar to those shown in Coe et al. (2019) at epochs earlier than MJD 57700 (a few hundreds of days before periastron). After the periastron passage, all the $\mathrm{H} \alpha$ parameters show fluctuations, and after $\sim 50$ days they slowly and smoothly recover to reach values similar to those before periastron. We interpret this as disruptions caused by the tidal interaction with the compact object. Another difference is that while, for PSR B1259-63, the increase in the equivalent width is steeper than the decrease, in PSR J2032+4127 we find the opposite behaviour. Both PSR B1259-63 and PSR J2032+4127 show evidence of a tilted disc with respect to the orbital plane. The main difference between them is that the former one has a bigger, denser, and very stable disc (Chernyakova et al. 2015) while disc in the latter is smaller and unstable (Coe et al. 2019). Since in both cases the pulsar crosses the disc near periastron the most dramatic effects seen in PSR J2032+4127 can, therefore, be interpreted as a consequence of the properties of its circumstellar disc. A comparison between how the pulsar disrupts a lower density and a higher density circumstellar disc can be seen in the SPH modelling shown in fig. 1 \& 2 of Takata et al. (2012). There the authors compare, for PSR B1259-63, the pulsar approaching the star/circumstellar disc for a base density of $\rho_{0}=10^{-11}$ and $10^{-9} \mathrm{~g} \mathrm{~cm}^{-3}$.

The maxima of the (absolute value) equivalent width of the $\mathrm{H} \alpha$ line are offset from the peaks of X-ray lightcurve (see Fig. 4, right panel). Assuming that the X-ray flux maxima correspond to the moments of the plane of the disccrossing, we conclude that the disc has a maximal size ((see e.g. Grundstrom \& Gies 2006)) significantly before and after disc crossing events. This can be understood, if a few competing processes take place in this system, e.g. star-pulsar tidal interactions (increasing disc size and build-up rate, as the pulsar approaches the periastron) and perturbations of the disc by the pulsar wind (continuous destroyment of the disc which leads to gradual decreasing of its size, e.g. sharp cutting the disc at distances higher than pulsar-star separation, see e.g. (Monageng et al. 2017; Coe et al. 2019)). Note that in Takata et al. (2017) it was demonstrated that if the base density is less than $\rho_{0}<10^{-10} \mathrm{~g} / \mathrm{cm}^{3}$, the pulsar wind can strip off an outer part of the Be disk, truncating it at a radius smaller than the pulsar orbit, and only the post-periastron X-ray flare may be related to disc crossing (Li et al. 2018).

The magnetic field in the emitting region typically considered for PSR B1259-63 is about $\sim 1$ G (see e.g. Chernyakova et al. 2015) which is comparable to values found here within the cooling scenario and somewhat higher than what is required for the acceleration scenario $(\sim 0.2 \mathrm{G})$. In terms of the energetics the spin-down luminosity of PSR B1259-63 exceeds that of PSR J2032+4127 by a factor of 5 (Romoli et al. 2017; Abeysekara et al. 2018), while the spatial scales of both systems at periastron are comparable ( $\sim 1 \mathrm{au})$. The higher magnetic field in PSR B1259-63 allows fast and more efficient cooling of the pulsar wind's electrons via synchrotron losses at timescales shorter or comparable to PSR J2032+4127. Within the cooling scenario this should lead to a similar formation of a low-energy tail in the spectrum of electrons. The characteristic tail's slope of $\Gamma_{e}=-2$ should result in an X-rays slope of $\Gamma \sim-1.5$ during the periods of disc crossing. Contrary, significant spectral softening (up to photon X-ray slope $\sim-2$ ) was observed during these periods (Chernyakova et al. 2015). Such a discrepancy is challenging to explain within the "cooling scenario", however it could be understood within "acceleration scenaio".

Fig. 6 (right panel) illustrates how different values of the magnetic field in the emitting region modify the final electron spectrum, for an initially injected cut-off powerlaw (with the slope of -2 ) electron spectrum in the "acceleration scenario". Namely, the increased value of the magnetic field leads to a significant softening of the spectrum at energies comparable to the injection energy $(\sim 0.1 \mathrm{TeV})$. The electron spectrum is modified in a similar way for a constant magnetic field but for longer electron escape times.

Within the "acceleration scenario" significant softening of the electrons' spectrum (and consequent softening of X-rays) during the disc crossing in PSR B1259-63 can be understood if the emitting region approaches the pulsar (magnetic field in the region increases) and/or escape time from the system significantly increase when the pulsar enters dense disc regions. The weaker $\mathrm{H} \alpha$ emission line observed in PSR J2032+4127 suggests that the disc is smaller than in in PSR B1259-63. This means that during the whole periastron passage, PSR J2032+4127 remains in a lower-density regions (than in PSR B1259-63) which results in shorter escape times and the magnetic field in the emitting region remains relatively low. This results in a hard X-ray slope of PSR J2032+4127 system during the whole periastron passage.

\section{CONCLUSIONS}

In this paper we analyse the results of multi-wavelength observation of PSR J2032+4127 as it is approaching and passing by its periastron in 2017. The observations of the $\mathrm{H} \alpha$ line show a clear variability of the Be star disc, both in the change in equivalent width, the line separation and the $\mathrm{V} / \mathrm{R}$ profile. X-ray emission from the system is also highly variable, increasing as the pulsar approaches periastron, but with a sharp minimum around the periastron itself. A clear link between the X-ray emission and the state of the Be star disc is demonstrated by the fact the X-ray spectral index changes at the same moment as the $\mathrm{H} \alpha$ equivalent width peaks.

The assumption that the disc of the Be star is inclined to the orbital plane allows the observed high energy variability to be explained in a natural way. In the proposed model X-ray and TeV emission observed from PSR J2032+4127 originate from synchrotron and inverse Compton processes, respectively. The observed spectrum from the source can be understood within the "cooling" or the "acceleration" scenario which relies on cooling/acceleration of monoenergetic electrons of pulsar wind, see Sec. 3.2.1 for the details. While both scenarios are able to satisfactory describe the SED of PSR J2032+4127 system, we argue that "acceleration scenario" seems to be preferable for other gamma-ray binaries, e.g. PSR B1259-63 and thus is natural for PSR J2032+4127 as well. 


\section{ACKNOWLEDGEMENTS}

The authors acknowledge support by the state of BadenWürttemberg through bwHPC. This work was supported by DFG through the grant MA $7807 / 2-1$, partially supported by the Spanish Government under grant PGC2018093741-B-C21 (MICIU/AEI/FEDER, UE), and the Russian Foundation for Basic Research project 17-52-80139 BRICS-a (SST). This work made use of data supplied by the UK Swift Science Data Centre at the University of Leicester. We acknowledge the use of public data from the Swift data archive and thanks the entire Swift team for accepting and planning Target-of-Opportunity requests. We also acknowledge funding to support our NOT observations from the Finnish Centre for Astronomy with ESO (FINCA), University of Turku, Finland (Academy of Finland grant nr 306531). The authors wish to acknowledge the DJEI/DES/SFI/HEA Irish Centre for High-End Computing (ICHEC) for the provision of computational facilities and support. We would also like to acknowledge networking support by the COST Actions CA16214 and CA16104.

\section{REFERENCES}

Abdo A. A. et al., 2009, Science, 325, 840

Abeysekara A. U. et al., 2018, ApJ, 867, L19

Aharonian F. et al., 2005, A\&A, 431, 197

Aharonian F. A., Atoyan A. M., 1981, Ap\&SS, 79, 321

Aharonian F. A., Kelner S. R., Prosekin A. Y., 2010, Phys. Rev. D, 82, 043002

Aliu E. et al., 2014, ApJ, 783, 16

Bednarek W., Banasiński P., Sitarek J., 2018, Journal of

Physics G Nuclear Physics, 45, 015201

Bell A. R., 2013, Astroparticle Physics, 43, 56

Blumenthal G. R., Gould R. J., 1970, Reviews of Modern Physics, 42, 237

Brown R. O., Coe M. J., Ho W. C. G., Okazaki A. T., 2019, MNRAS, 488, 387

Camilo F. et al., 2009, ApJ, 705, 1

Chernyakova M. et al., 2019, A\&A, 631, A177

Chernyakova M., Neronov A., Lutovinov A., Rodriguez J., Johnston S., 2006, MNRAS, 367, 1201

Chernyakova M. et al., 2015, MNRAS, 454, 1358

Coe M. J. et al., 2019, MNRAS, 485, 1864

Corbet R. H. D. et al., 2019, ApJ, 884, 93

Dubus G., 2013, A\&A Rev., 21, 64

Grundstrom E. D., Gies D. R., 2006, ApJ, 651, L53

Hanuschik R. W., 1989, Ap\&SS, 161, 61

Ho W. C. G., Ng C. Y., Lyne A. G., Stappers B. W., Coe M. J., Halpern J. P., Johnson T. J., Steele I. A., 2017, MNRAS, 464, 1211

Hummel W., Hanuschik R. W., 1997, A\&A, 320, 852

Johnston S., Lyne A. G., Manchester R. N., Kniffen D. A., D'Amico N., Lim J., Ashworth M., 1992, MNRAS, 255, 401

Jones F. C., 1994, ApJS, 90, 561

Khangulyan D., Aharonian F., Bosch-Ramon V., 2008, MNRAS, 383, 467

Khangulyan D., Aharonian F. A., Kelner S. R., 2014, ApJ, 783,100

Klement R. et al., 2017, A\&A, 601, A74
Kolka I., Eenmäe T., Laur J., Aret A., 2017, Research Notes of the American Astronomical Society, 1, 37

Li K. L., Takata J., Ng C. W., Kong A. K. H., Tam P. H. T., Hui C. Y., Cheng K. S., 2018, ApJ, 857, 123

Lyne A. G., Stappers B. W., Keith M. J., Ray P. S., Kerr M., Camilo F., Johnson T. J., 2015, MNRAS, 451, 581

Malyshev D., Chernyakova M., Santangelo A., Pühlhofer G., 2017, arXiv e-prints

Melatos A., Johnston S., Melrose D. B., 1995, MNRAS, 275,381

Monageng I. M., McBride V. A., Coe M. J., Steele I. A., Reig P., 2017, MNRAS, 464, 572

Ng C. Y. et al., 2019, ApJ, 880, 147

Okazaki A. T., Bate M. R., Ogilvie G. I., Pringle J. E., 2002, MNRAS, 337, 967

Pal P. S., Tam P. H. T., Cui Y., Li K. L., Kong A. K. H., Güngör C., 2019, ApJ, 882, 25

Panoglou D., Carciofi A. C., Vieira R. G., Cyr I. H., Jones C. E., Okazaki A. T., Rivinius T., 2016, MNRAS, 461, 2616

Reig P., Nersesian A., Zezas A., Gkouvelis L., Coe M. J., 2016, A\&A, 590, A122

Rivinius T., Carciofi A. C., Martayan C., 2013, A\&A Rev., 21,69

Romoli C., Bordas P., Mariaud C., Murach T., H. E. S. S. Collaboration, 2017, International Cosmic Ray Conference, 301, 675

Rouco Escorial A., Hernández Santisteban J. V., Echevarría J., Wijnands R., Page D., Degenaar N., 2019, Research Notes of the American Astronomical Society, 3, 31

Salas J., Maíz Apellániz J., Gamen R. C., Barba R. H., Sota A., Sánchez-Bermúdez J., Alfaro E. J., 2013, The Astronomer's Telegram, 5571, 1

Takata J. et al., 2012, ApJ, 750, 70

Takata J., Tam P. H. T., Ng C. W., Li K. L., Kong A. K. H., Hui C. Y., Cheng K. S., 2017, ApJ, 836, 241

Telting J. H., Heemskerk M. H. M., Henrichs H. F., Savonije G. J., 1994, A\&A, 288, 558

The Fermi-LAT collaboration, 2019, arXiv e-prints, arXiv:1902.10045

Zabalza V., 2015, ArXiv e-prints

Zamanov R. K., Reig P., Martí J., Coe M. J., Fabregat J., Tomov N. A., Valchev T., 2001, A\&A, 367, 884 